\title{
Student Spectrophotometry of the Planets: What Worked and What Did Not
}

\author{
Richard Berry ${ }^{1 *}$
}

\section{Abstract}

In summer 2016 I worked with two high-school students on a relatively advanced spectrophotometry project at the University of Oregon's Pine Mountain Observatory. My plan had been to use an ALPY 600 spectrograph on a $65 \mathrm{~mm}$ refractor to do an introductory survey of stellar spectral types. But when the small telescope's mount died, I asked the students what they wanted to do. They said: Let's do planets! Since Pine Mountain Observatory's 24-inch $\mathrm{f} / 13$ Cassegrain was available, we put the ALPY on the 24-inch, and shot spectra of Jupiter, Mars, Saturn, Titan, Uranus, Neptune, Triton, and the Moon. Everything worked perfectly (the success part!), but understanding what the data meant required more chemistry, physics, math, and software skills than the students could begin to absorb (i.e., not a success).

${ }^{1}$ Alpaca Meadows Observatory, Lyons, Oregon

*Corresponding author: rberry@wvi.com

\section{Introduction}

We will hear a lot of success stories at this meeting. But I'm going to tell you about an experience that I had about a year ago that was a good part of my motivation for coming to this meeting. Let me paint a picture: we're at the University of Oregon's Pine Mountain Observatory, at 6,500 feet elevation, doing a four-day summer astronomy workshop for high-school students. The students stayed in an adjacent campground an easy walk from the observatory.

The workshop was put on by six amateur astronomers who served as mentors. The six of us who did this felt the need to pay back the experiences we'd had in the 50s and 60s that got us interested in astronomy or interested in computers, and ultimately led us to science-related careers. The projects were based on what those of us involved were interested in, or into, or excited by. Each of us mentored two to four high-schoolers; two were assigned to me. Projects included taking pretty pictures, asteroid orbit determination, and measuring scintillation noise. Since I had been playing with a new spectrograph, I planned to introduce my two students to stellar spectroscopy.

Although Pine Mountain Observatory has a 24-inch f/13 Boller and Chivens Cassegrain reflector, all of the mentors brought their own equipment, much of it high-end amateur gear. For example, the guy doing asteroid orbits brought a heavy-duty AstroPhysics mount with a $120 \mathrm{~mm}$ Takahashi refractor. The different projects were spread along the walkway between the 24-inch dome and the 32-inch dome (the 32-inch was not functioning). We had four beautifully clear and moonless nights in a row.

Each group of students was expected to give an afternoon presentation on their activities on the last day of the workshop. Mentors gave talks in the mornings. In addition to producing an "instant talk," some students expected to use the data we collected in a senior-year project at their high schools. The younger of my two was just entering tenth grade, but the older would be in her senior year that fall. 
My plan - to introduce stellar spectroscopy - was that we would collect spectra from a list of "Oh Be A Fine Girl Kiss Me" stars so the kids would see what is involved in taking astronomical data. While still under the stars, we would take a quick look at each spectrum, and it would be clear that the spectra of stars vary. We could discuss what we were seeing, and during the day I hoped that we would have time to reduce the data and then attempt to arrange the spectra in some logical order, just as astronomers did in the early 20th Century.

The equipment I brought was rather modest: an ALPY 600 spectrograph mounted on a $65 \mathrm{~mm}$ f/6.5 refractor and Celestron AVX mounting. The ALPY is a compact design that provides a spectral resolution of 500 over a range from 375 to 750 nanometers - the whole visual spectrum. The ALPY has two CCD cameras: one to capture the spectra and one to view the slit of the spectrograph. With large telescopes, seeing enlarges the star images, so only a fraction of a star's light enters the spectrograph. The beauty of using a spectrograph on a small refractor is that the refractor forms tiny, clean star images, so nearly all the light from a star goes into the ALPY's 23-micron slit. Relative to expectations, the small system outperforms larger ones because all the light gets used. When you are guiding, you can see that the entire star image disappears into the slit.

We set up the telescope, mount, and spectrograph on a graveled area, and located the students and a computer with control software on a picnic table beside it. Once everything was working —at least in theory - the mount, spectroscope, and CCD cameras could be run remotely from the computer ten feet away. We got started quickly enough; we soon had spectra of bright stars of different types. I sat with them and made sure everything kept working. I may have helped them too much by finding the stars for them, but they quickly picked up the skills for centering stars, guiding on the slit, starting exposures, and saving spectra to the hard drive.

Well, it got cold on the mountain. No matter what you tell high-school kids, they don't believe the

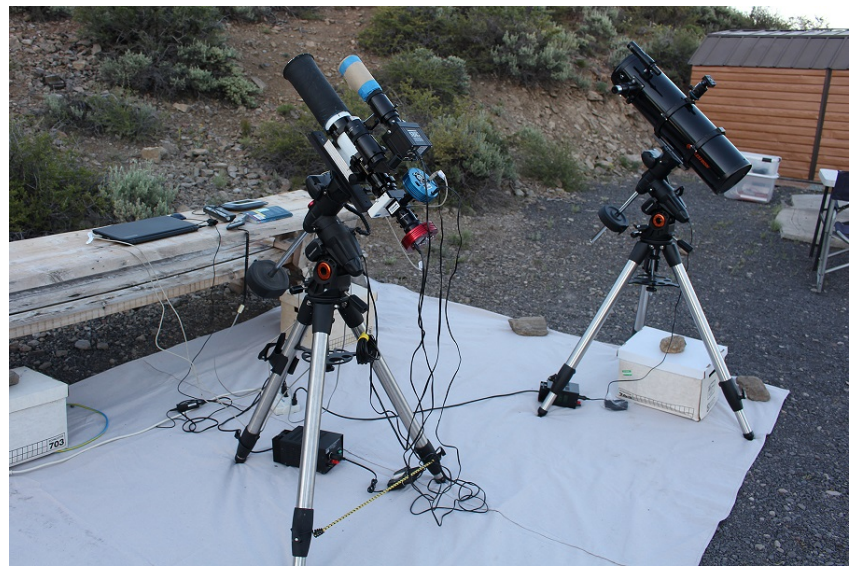

Figure 1. Our original observing plan employed a 65 mm refractor with a Shelyak ALPY 600 spectrograph. Observers were seated at a picnic table on the north side of the telescope. The second instrument was a small Newtonian telescope for recreational visual observing during longer exposures.

temperature might drop to 32 degrees with a 15 mile an hour wind in the middle of summer. So they were freezing to death, and they were not used to staying up late at night. By midnight they were miserable and their brains had stopped working, so we called it quits.

The next morning I found the students had already begun to prepare their presentation. They had mined Wikipedia for stuff about stars, and the question they had for me was, "What is our hypothesis?" The Scientific Method, they had been taught, involved framing a hypothesis that we would test using our observations. We had a long discussion during which I learned about the older student's project from the previous year that involved the dissolution of coins in various acids and solvents. Collecting an O-B-A-F-G-K-M sequence did not fit into a scientific method scheme, so I suggested we try something different: "Let's look at M stars, the red ones with the bumpy spectra. We can look for stars with different amounts of carbon, oxygen, and nitrogen." It was not a very good idea, but I hoped we could put together something that would be suitable for her senior project.

It proved tough to come up with a list of suitable 


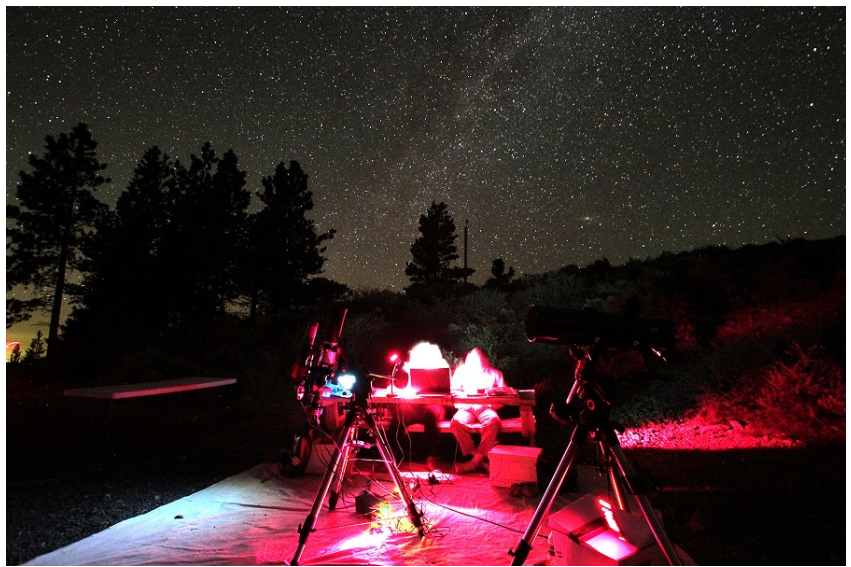

Figure 2. Even when the screens were reduced to their lowest intensity, the laptop computer screens effectively blinded the observers controlling the CCD spectrum and guide camera. The night was clear and became quite chilly, so the student observers, who sat while operating the CCD camera from the computer, became very cold.

type M stars. They needed to be oddballs, and they needed to be reasonably bright for the small aperture of the telescope. The evening started fairly well. After the first several stars, I left the kids gathering spectra and went off to see what the other groups were up to. When I came back, I noticed that the telescope was pointing far from the nominal target. A quick check showed that the spectra they had taken were not $\mathrm{M}$ stars at all. For some reason, the mount was pointing at anything but the target stars. Added to that, it was cold again. We gave up some time after midnight.

\section{What Did Work}

The next morning I took a walk with the students. I said, "You guys, we've got nothing ... we don't have a hypothesis with this project. We've collected some spectra of stars around the sky. It's like a collection of leaves from different trees. We don't know enough about trees or leaves to make a hypothesis."

"However," I said, "We have two more nights, so at least we ought to have some fun. What would you like to do?" And they said, "We want to do some planets!" Mars, Jupiter, and Saturn had been temptingly lined up across the southern sky that July, shining brilliantly, and, for most people, planets are more familiar and comprehensible than stars. So I said. "Okay. I'll see whether we can hang our spectrograph on the 24-inch telescope, and I don't think anyone else is using it. It will be fun. Besides, it'll be warmer inside the dome than it is outside in the breeze."

So we hung the tiny ALPY spectrograph on the rear of the huge 24-inch Cassegrain, and strung the control wires to the students sitting at a folding table beneath the telescope. As darkness fell, we aimed the big telescope at Jupiter. With its 8 meter focal length, Jupiter was fully half the height of the slit. Although the seeing was not great, and cloud bands were visible in the guide camera image. We carefully aligned the ALPY so the slit ran along the Jovian equator, and then shot a series of 10 -second exposures. That's when the serendipity kicked in: three of the Jovian satellites were visible. We grabbed a 300-second exposure of each one, and then moved the telescope over to Mars. Since the polar caps were visible, we captured a series of 5-second spectra from the polar caps as well as the east and west limbs of the planet. I explained that I did not know whether our spectra would show any differences in the light reflected from the planet's red deserts and icy polar regions, but if we took the spectra, we would find out.

Saturn proved to be a delight. With the slit running along the equatorial axis, spectra of the ball of the planet were flanked by spectra of the rings. Each Saturn exposure required 60 seconds. All three of the parallel spectra showed Fraunhofer lines, but only the ball of the planet showed obvious dark absorption bands. More serendipity: there was Titan, a satellite with an atmosphere! Would we see methane there, too? The best thing that happened that night - at least it was fun for me occurred after we finished Saturn. The younger student had already conked out. I checked an ephemeris, and proposed to the older student, "Do you think you have the energy to wait for Neptune to come around? We've done two giant planets, Jupiter and Saturn, but Neptune is another giant 


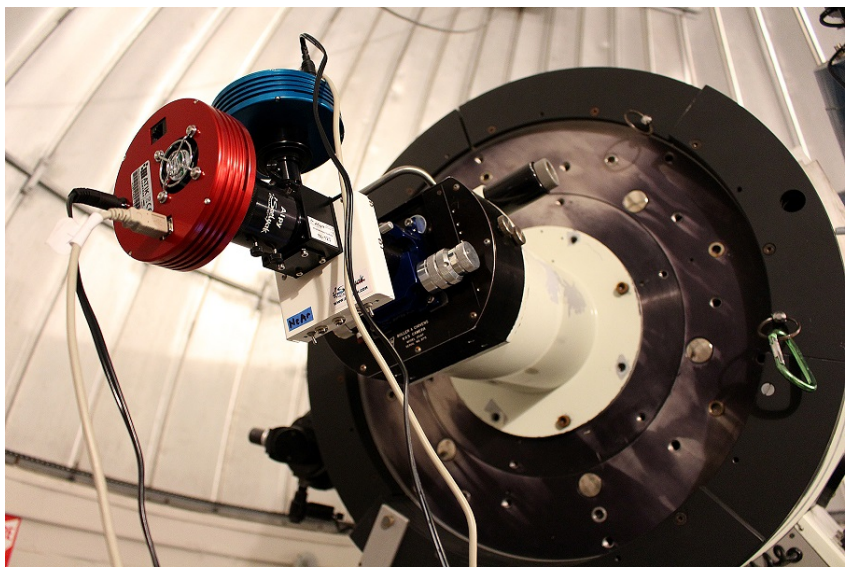

Figure 3. The compact ALPY 600 spectrograph worked extremely well on Pine Mountain Observatory's 24-inch f/13 Cassegrain telescope. All functions of the ALPY were controlled "by wire" by the observers seated at a table below the telescope.

planet." Would it be like Jupiter and Saturn, or would it be different?

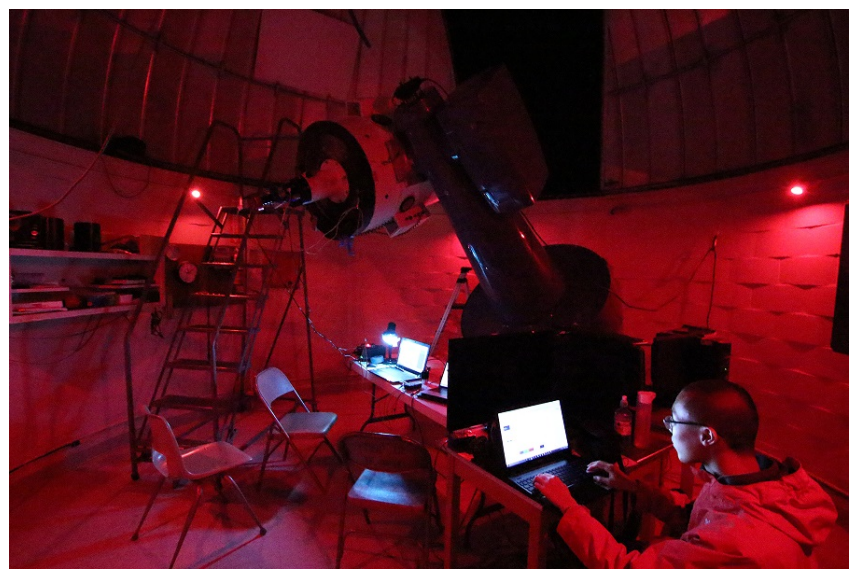

Figure 4. The younger student, seated at his computer, attempted to process spectra in real time. With more compatible computers and software, this could have worked very nicely.

"Well, maybe," she said. "It'll be up in two hours, so why don't we do some calibration spectra on stars?" So the two hours went by quickly as we gathered spectra of some type A stars that were high in the sky. When Neptune came around, we did ten five-minute exposures and got some nice Neptune data. We even tried one of Triton, the eighth-magnitude satellite of Neptune. Then I sprung it on her: "In another hour, we can get

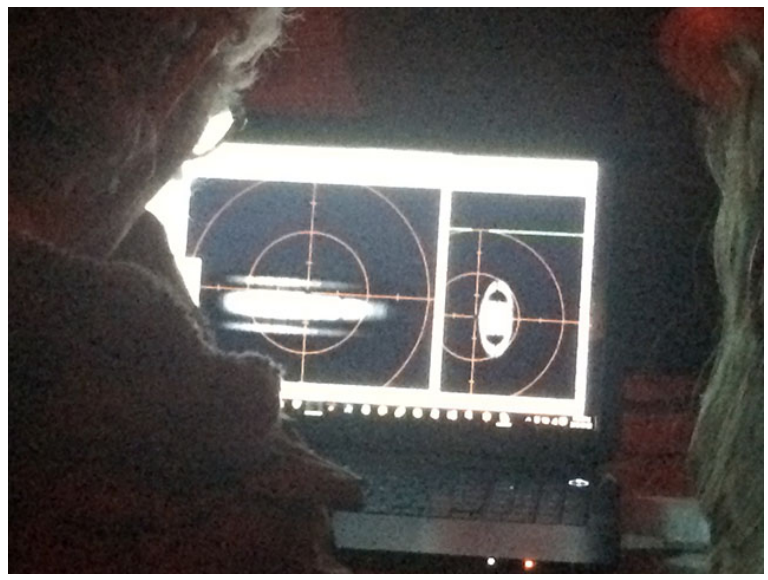

Figure 5. The screen of the computer we used to control the spectrograph displayed images from the spectrum camera (on the left) and the slit-monitor and guide camera (on the right). The students used the telescope control paddle to center and guide images of the planets.

Uranus," and when it rose we spent another hour taking 5-minute exposures of Uranus' spectrum.

By now, dawn was coming and the sky had begun to brighten. I looked out the dome slit and I saw that the Moon was coming up. "Look, we can get a calibration spectrum that will fill the entire slit, and it's a solar spectrum from an airless body, modified only by passage through the Earth's atmosphere." We pointed the telescope at the Moon as soon as it cleared the dome wall, and got some fine lunar spectra for calibration.

We parked the telescope and closed the dome a little before the Sun came up. We had ended up with beautiful spectra of Jupiter, Saturn, Uranus, and Neptune, Mars, and the Moon - as well as three of the Galilean satellites of Jupiter, and Saturn's moon Titan. We had beautiful data. We had methane and ammonia bands on the giant planets, and when we had done Mars we also had the equatorial regions versus the polar caps. The following night, the last night, we shot more images of Saturn in the early evening and left the CCDs taking bias frames dark frames until dawn.

Because we undertook making planetary spectra in the spirit of fun, the students seemed to enjoy the process much more than they had the previous 
nights of taking stellar spectra for a scientific presentation. It helped that it was relatively warm in the dome. Also, I think the 24-inch was closer to their conception of "telescope," and the planets were more varied and "sexy" than stars. Both students mastered focusing the image using the in/out focus motor, centering objects on the slit, guiding to keep an object on the slit, setting exposure times, taking exposures, saving exposures, as well as keeping a written log of the objects, exposures, and context data. We had a lot of time in the dimly lit dome to discuss what we were doing, and made informal scribbles on a legal pad. The younger student fell asleep at midnight, but before he did, he had taken his turns guiding the telescope. I had enough time to find out how much (and how little) they already knew, and how we might be able to shape their presentation to report what we had accomplished.

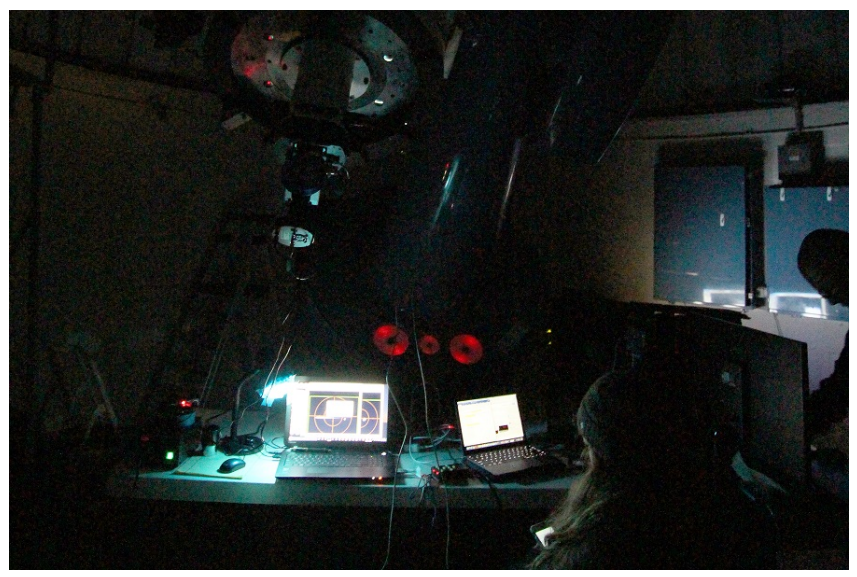

Figure 6. In addition to starting exposures and guiding, the students recorded "meta data" on their personal computers, and began work on the presentation even as we were collecting data. This photo was taken around 4:00 a.m., toward the end of a long night of taking spectra.

I won't delve into the science of spectrophotometry, except to explain, in as few words as possible, what we got. The Sun illuminates the planets, and its spectrum is the classic Fraunhofer spectrum, full of spectral absorption lines. When sunlight reflects from a body in space, the body modifies the solar spectrum. The straight reflectance spectrum of
Saturn combines the effects of the solar spectrum with the reflectance spectrum of Saturn. The gas giant planets Jupiter and Saturn, and the water giant planets, Uranus and Neptune, absorb energy due to the molecular absorption bands of methane and possibly ammonia. We were fortunate in getting a beautiful set of reflectance spectra of the Moon, which has no atmosphere, so its spectrum consists of the sunlight that is only slightly yellowed by the minerals in the lunar surface.

When you divide Saturn's spectrum by the lunar spectrum, the Fraunhofer lines common to both divide out and - BINGO - you see the absorption lines standing out loud and clear on the ball of the planet, but no such features on the rings. The younger student quickly became adept at using ImageJ. When he asked me how to extract a one-dimensional profile from a spectrum, I told him I did not know, but it was such a basic operation that ImageJ was sure to do it. He figured it out on his own in about five minutes.

\section{What Didn't Work}

Computers. I had a PC. They had Macs. Software? The program I had intended to use for reducing the spectra, Tom Fields' RSpec, did not at that time run on Macs. I like RSpec: it's easy to use, has a short learning curve, and it teaches as you learn to use it ${ }^{1}$ But there's nothing magic about extracting a one-dimensional profile from a two-dimensional image. I fished up and installed ImageJ, a flexible image-processing program that ran on their Macs as well as on my PC. The younger student was clearly impressed with its capabilities. So that turned out okay.

The students knew all about Google Drive; I had only heard about it. I logged in and fifteen minutes later we were in business. We used Google Drive to move our data from my computer to theirs, and various Google apps to prepare their presentation. Although the Internet connection on the mountain was slow, it was fast enough to let us locate and

\footnotetext{
${ }^{1}$ For more about RSpec software, see https://www. rspec-astro.com.
} 
download ImageJ, and it allowed us to work effectively with Google apps. It also gave the students access to Wikipedia, which they considered essential to preparing the presentation expected of them.

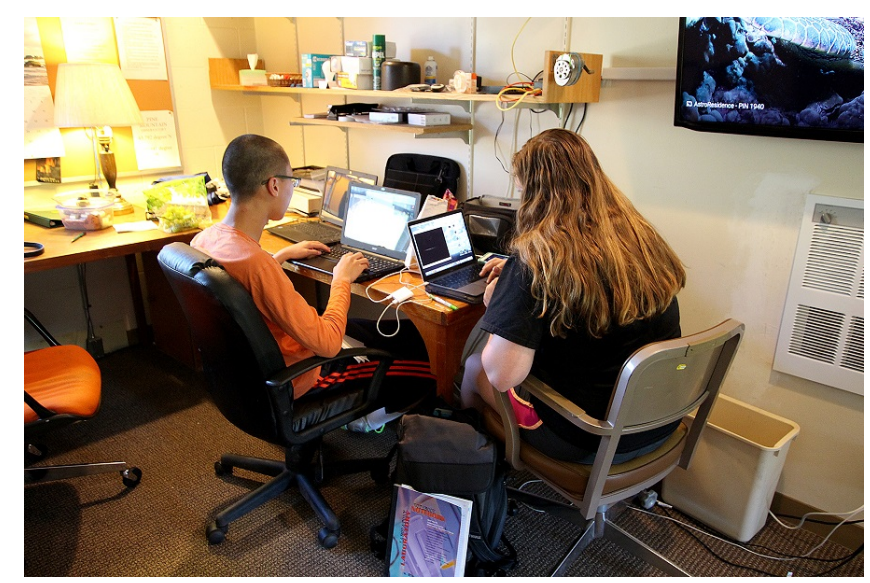

Figure 7. With the help of Google Docs and Google Show, the students reduced the data and prepared their presentation. The younger student, on the left, using ImageJ, produced intensity profiles, while the older student researched spectroscopy and prepared slides.

That, in fact, highlights the mismatch in thinking between the students and me. The students felt pressured to get the "right" results. They were acutely aware they would be speaking to other bright high-school kids, to their teachers, and to the other mentors. But I had gone out with the attitude: I'm not sure what we're going to get with these planets. I knew in general what we would get - methane in the giant planet atmospheres would surely be unmissable — but I had only a rough idea what the exposures would be required for planets on the big telescope, or how good our data would be. Basically, I was improvising and learning as we went. We would shoot a spectrum, measure the pixel values in it, and try another exposure. That initially freaked them out. They wanted me to be the expert; someone who could guide them safely through a mysterious process, someone who was going to tell them what was going to happen next.

I think that the younger student understood the idea first: try an exposure, measure the result, adjust the

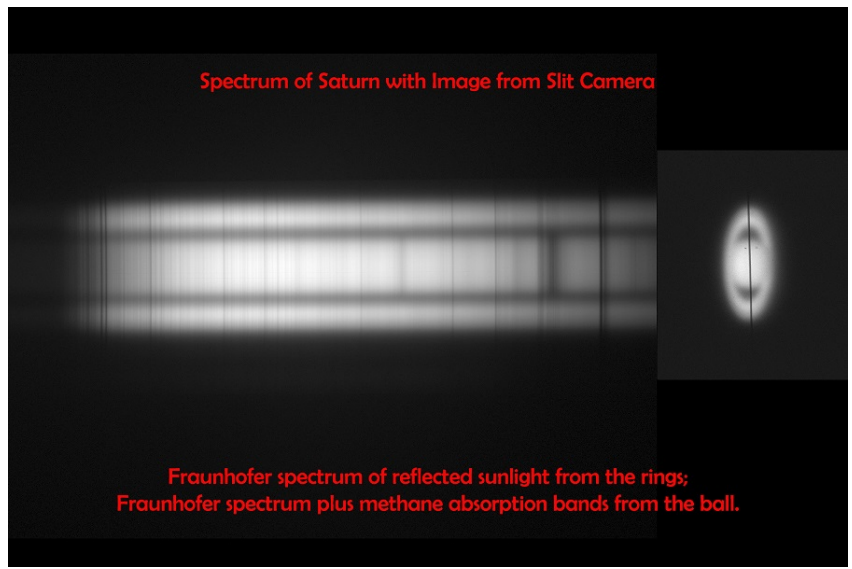

Figure 8. Saturn demonstrates reflectance spectroscopy of the planets. At right, the image of Saturn rests on the slit of the spectrograph, while the spectrum appears on the left. The solar Fraunhofer spectrum appears in the spectrum of the ball and the rings of the planet, but methane absorption bands appear only in the spectrum of the ball.

exposure, try again. The enormous size of the planetary images from the big telescope - two millimeters across Saturn's ring system — meant that only a tiny fraction of the planet's light entered the 23-micron slit of the spectrograph. It turned out that our Saturn spectra required 60 seconds, but we needed only 300 seconds for its very much dimmer satellite Titan, courtesy of Titan's compact star-like image. That was a nice surprise!

In a school setting, you'd have a month or so to teach students about light, what a spectrum is, etc.: all that background needed to comprehend spectroscopy. In a four-day workshop setting, with one student going into tenth grade and one going into twelfth grade, they were unsure in their knowledge of the solar system and which planets were which. Astronomy is not taught in Oregon schools, but in the popular media, characters routinely fly off to planets and galaxies with equal ease. Given what they see and hear, it's not hard to understand why basic astronomy is confusing. 


\section{What They Learned}

It's difficult to assess — and partly speculation on my part — what the students actually learned from their experience at Pine Mountain Observatory. Based on the presentation they gave for the other students, their teachers, and the other mentors, as well as on comments they made at the time, I offer the following:

- It gets cold on a mountain at night.

- The big telescope was fun to use!

- Data are precious.

- When you try to find a star, they all look alike.

- Five-minute exposures take forever.

- You must guide to keep Saturn on the slit.

- Data does not analyze itself by itself.

- Spectra are smeary streaks.

- Spectrum data is a bumpy line.

- Some of the bumps mean Jupiter has gas.

"Some of the bumps mean Jupiter has gas." Well, you have to admit that although it may sound simplistic, that statement is true enough. So in some respects, they did grasp the basic rationale for what we were doing. The younger student, who did the bulk of the work extracting spectral curves from the spectrum images, may have gained a better understanding, but the older student prepared most of their presentation. What they didn't get in the presentation is the big picture. In their presentation, they did not explain that the Sun has a spectrum with Fraunhofer lines in it, that the Sun shines on the planet, the planet modifies the spectrum when reflects the sunlight, and that, we, viewing the planet through the Earth's atmosphere, can, through clever manipulation of the spectral data that we took, deduce something about the properties of the planet's surface.
They did not even come away from the experience, I think, with a sense of joy from getting out there and looking closely at some aspect of the real Universe. They were trying to force-fit their "doing science" experience into their understanding of the scientific method, with a hypothesis and so on. They were aware that astronomers already have textbook answers, and they were concerned about getting the right answers. During those hours in the 24-inch dome, I stressed, "There is no right answer. What we observe here and now is what is actually out there." I don't think they really understood that what we do as astronomers and observers informs the textbooks, not the other way around.

\section{Afterword}

I closed my talk at the RTSRE conference saying, "If we're going to do these summer research projects more effectively, we must adopt better methods and better thinking. In the six months since that time, I have thought about this a good deal. The very simplest change would be to meet with the students two or three weeks ahead of the workshop, and spend a day talking through what the projects would be and what doing them would involve. Given a basic grounding, the opportunity to ask questions, and some time to do some on-line research, they could arrive both prepared and ready for the direct experience.

It may be difficult, but I think we owe it to potential scientists to tell them that the scientific method is a high-level description of a process that working scientists usually honor only in the breach. We work, we think, we discuss, we make observations, we get inspirations - and afterward we write it up as if it had been a systematic process. The reality is that we muck around exploring the territory, making observations just to see what's going on. In an observational science like astronomy, this often occurs when we get new instruments or observing tools. This open pattern of exploration is what I planned to do with the students and the spectrograph: "Let's look at a bunch of stars and see what is out there." Of course, I wanted the spectra we took to include every type 


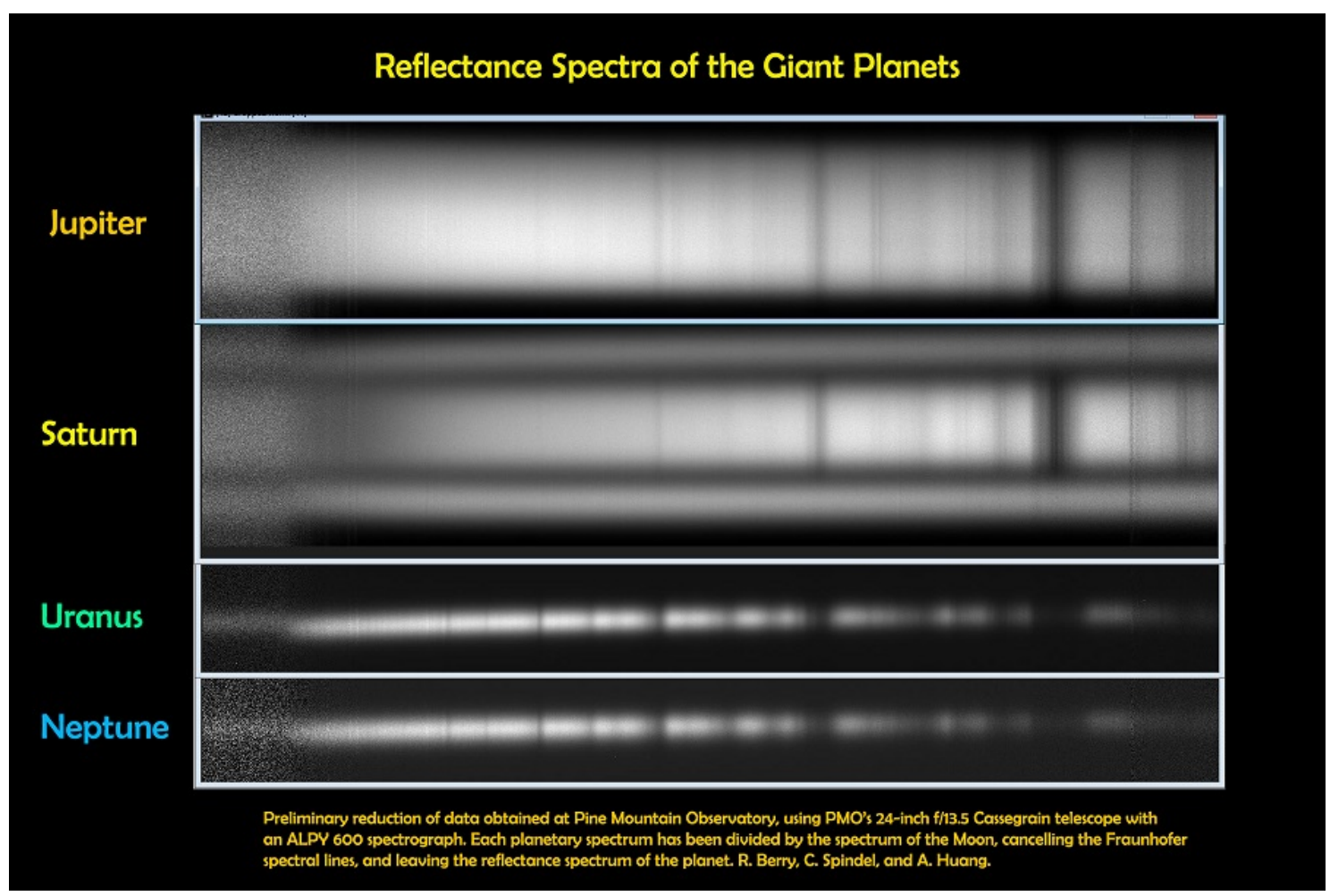

Figure 9. Our spectra from Pine Mountain Observatory show gas absorption bands for the giant planets. In each case, the raw reflectance spectrum has been calibrated and then divided by the reflectance spectrum of the Moon, thereby removing the solar Fraunhofer lines. Jupiter shows both methane and ammonia bands, Saturn's ball shows methane and weak ammonia bands while the rings display no absorptions. The spectra of Uranus and Neptune show very deep methane absorption.

of star, not just the common A, F, G, and $\mathrm{K}$ stars, so I had salted the list with the relatively rare $\mathrm{O}, \mathrm{B}$, and M-type stars.

Once we think we understand the territory, scientists tend to standardize methods of collecting data. That is more or less what the students and I did with our spectra of the planets. Instead of searching, we followed an observing protocol to make scientific measurements. Much of ordinary science consists of doing this type of bread-and-butter observation; filling in gaps, populating databases, and keeping our eyes open for anything out of the ordinary. A formal scientific method is far from our minds most days; we're working inside a well-established body of knowledge, decorating the walls with more stuff that fits the prevailing meme.

So when does the scientific method come into play? In my experience, we drag it out and apply it when the going gets sticky. We have observations that don't fit. We have a hunch that might explain what's going on. The purely creative process breaks down, and we start writing things down. We make a list of hunches. We run gedanken experiments. We do the math. We need access to a bigger telescope, so we write a proposal to the time allocation committee, and have to get everything down on paper. I think that when we explain how science really works - a creative endeavor that is both loosy-goosy and extremely demanding -we'll find that more students will get excited and want to join in playing this grand game.

It's important to point out that I'm a 70-year-old guy who has been playing with telescopes and gadgets for 58+ years, so I have a lot of experience to fall back on when things go awry. For example, I had no qualms about operating PMO's 24-inch Cassegrain telescope with ten minutes instruction from the observatory's tech support guy. I knew 
that a nominally $\mathrm{f} / 5$ spectrograph would work happily on the f/13 telescope, and I knew enough to know that a focal-ratio mismatch could have been a problem. I knew enough to get the telescope focused despite a quirky focus motor. I've had enough experience with both PCs and Macs to recognize that we could use Image J on both, and was delighted to discover the existence of AstroImageJ. Finally, I'm not in the teaching profession. I'm having fun doing this. I have no skin in this game. I can be wrong and it doesn't matter.

I was greatly intrigued by the Freed and Genet "communities of practice" concept. Although not intended as a teaching environment, between my junior and senior year in high school I was a summer "observer" at Yale University's Bethany Station near New Haven. My job was to operate a 20-inch telescope with a four-channel

spectrophotometer. This meant running all systems from rewinding the weight-driven clock drive to servicing the photomultiplier tube coolant loop each night with dry ice. I was in hog heaven! On clear nights we observed possible flare stars all night long. On cloudy nights we observed the dark current in the photomultiplier tubes all night long. During the days, I was free to annoy the graduate students operating radio telescopes observing Jovian decametric radiation (it had been recently found to be modulated by Io) with endless questions.

Since the RTSRE conference, I have had a very positive experience working with four undergraduate physics student from Portland Community College. The project was to recreate the Eddington Experiment of 1919 using modern amateur equipment during last summer's solar eclipse. Although their professor has no practical experience in observational astronomy, he is good at getting grants, and had spotted this project as a good one. I was recruited, in part, because my property and my observatory were nearly on the center line.

These students are "non-traditional" in different ways. Two are in their early 30 s, one in his late 30 s, and one is a home-schooled high-school-age student who has been taking college-level courses for several years. Four months before the eclipse, even before the RTSRE conference, we had set up a small telescope with a CCD camera on my property. Two students trained on that telescope and the other two trained with a portable telescope. We had more than a dozen sessions together. We worked through a large number of problems to be able to carry out high-precision astrometry with small telescopes. About three days before the eclipse, I pulled back and let the students work out the details between themselves. I could see they were a bit scared, but during the eclipse, when there would be no time to ask questions, they had to be self-sufficient. They captured excellent data —in focus and properly exposed — consisting of 23 eclipse images and 10 astrometric reference images. Our next challenge would be to reduce these images to determine whether the gravitational deflection predicted by Einstein's General Relativity agrees with the deflections we measured this summer. However, we had failed to consider how complex data reduction would prove to be. I proposed writing reduction software using Python's NumPy, SciPy, and AstroPy libraries — only to learn that not one of the students had any prior experience writing software, and at that point I was just beginning to learn Python. Not surprisingly, progress has been slow; a few key routines are now written and we have produced a few pretty charts. But the students have regular spring-semester classes, and the Eddington Experiment is a non-class activity that does not even offer them extra credit. I am hoping to rouse their interest this coming summer, to complete the data reduction.

My suspicion is that no amount of theory or classroom work could have prepared these students for this experiment as doing the grunt work and solving the practical problems we encountered. The "hands-off" nature of robotic telescopes worries me. Observational astronomy seems to require a certain "gut feel" for the costs and pitfalls of gathering actual data. You get cold. You get sleepy. A technician puts the wrong filter in the 


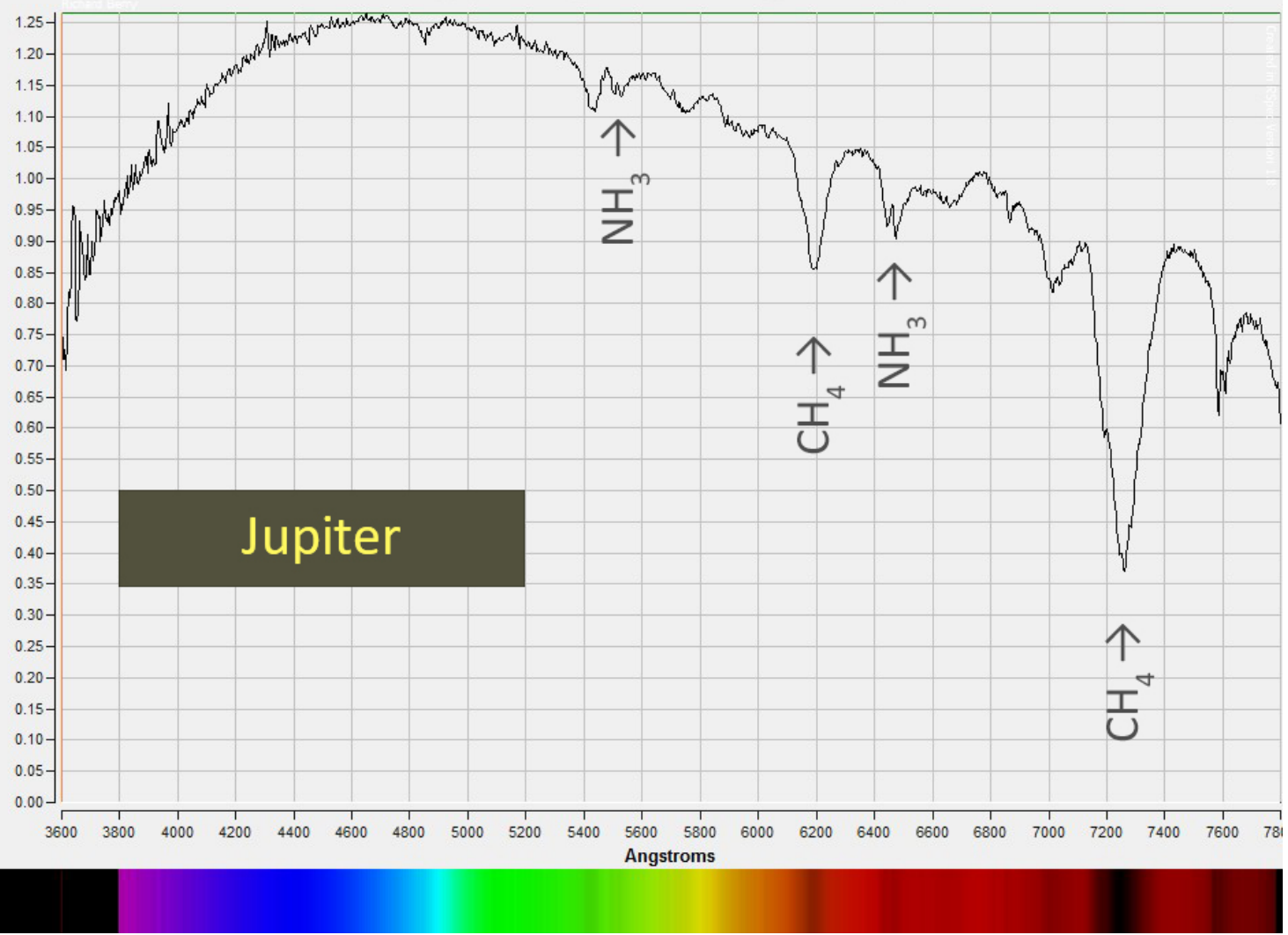

Figure 10. Jupiter divided by lunar spectrum.

filter wheel. You erase the wrong directory. Power supplies fail. Only after teachers and their students experience the good, the bad, and the ugly of working hands-on with the tools of their trade, can they properly love, appreciate, trust, and distrust a remote robotic telescope.

\section{Acknowledgements}

I would like to thank the Pine Mountain

Observatory, its Director, Scott Fisher, and Alton

Luken, Operations Manager, for opening the

Observatory and its facilities to volunteer educational activities. Pine Mountain Observatory, located 34 miles southeast of Bend, Oregon, is perched atop a mountain at an elevation of 6,300 feet. The observatory's location is well placed to make the most of the dark skies that the Eastern Oregon high desert provides. The observatory is operated by the University of Oregon, Department of Physics, under a special use permit from the Deschutes National Forest. The observatory's primary function is research and other astronomical observations, including basic and advanced scientific research. However education at all levels is also an important function and objective of the observatory. The current schedule of activities is located at https://pmo.uoregon.edu. 